\title{
A set of innovative immersed grating based spectrometer designs for METIS
}

Tibor Agócs ${ }^{\mathrm{a}^{*}}$, Ramon Navarro ${ }^{\mathrm{a}}$, Lars Venema ${ }^{\mathrm{b}}$, Aaldert H. van Amerongen ${ }^{\mathrm{c}}$, Paul J.J. Tol ${ }^{\mathrm{c}}$, Hedser van Brug ${ }^{\mathrm{d}}$, Bernhard R. Brandl ${ }^{\mathrm{e}}$, Frank Molster ${ }^{\mathrm{e}}$, Stephen Todd ${ }^{\mathrm{f}}$

${ }^{a}$ NOVA Optical Infrared Instrumentation Group at ASTRON, P.O. Box 2, 7990 AA, The Netherlands; ${ }^{\mathrm{b}}$ ASTRON, P.O. Box 2, 7990 AA Dwingeloo, The Netherlands; ${ }^{\mathrm{c}} \mathrm{SRON}$ Netherlands Institute for Space Research, Sorbonnelaan 2, 3584 CA, Utrecht, the Netherlands; ${ }^{\mathrm{d}} \mathrm{TNO}$ - Technical Sciences, Stieltjesweg 1, 2628 CK, Delft, the Netherlands; ${ }^{\circ}$ Leiden Observatory, Leiden University, P.O. Box 9513, 2300 RA Leiden, The Netherlands; ${ }^{f}$ UK Astronomy Technology Centre, Royal Observatory, Blackford Hill, Edinburgh, EH9 3HJ, UK

\begin{abstract}
We present innovative, immersed grating based optical designs for the SMO (Spectrograph Main Optics) module of the Mid-infrared E-ELT Imager and Spectrograph, METIS. The immersed grating allows a significant reduction of SMO volume compared to conventional echelle grating designs, because the diffraction takes place in high refractive index silicon. Additionally, using novel optimization techniques and technical solutions in silicon micromachining offered by the semiconductor industry, further improvements can be achieved. We show optical architectures based on compact, double-pass Three Mirror Anastigmat (TMA) designs, which appear advantageous in terms of one or several of the following: optical performance, reduction of volume, ease of manufacturing and testing. We explore optical designs, where the emphasis is put on manufacturability and we investigate optical solutions, where the ultimate goal is the highest possible optical performance. These novel, silicon immersed grating based design concepts are applicable for future earth and space based spectrometers.
\end{abstract}

Keywords:

Immersed grating, optical design, mid-infrared, astronomy, spectrograph, METIS, E-ELT, silicon

\section{INTRODUCTION}

The 'Mid-infrared ELT Imager and Spectrograph' (METIS) will be among the first generation of scientific instruments on the European Extremely Large Telescope (E-ELT), the only E-ELT instrument to cover the thermal/mid-infrared wavelength range from $3-14 \mu \mathrm{m}$. The METIS instrument baseline includes two main subsystems with both of them working at or near the diffraction limit. The diffraction limited imager at $\mathrm{L} / \mathrm{M}$ and $\mathrm{N}$ band with $20^{\prime \prime} \times 20^{\prime \prime}$ wide Field Of View (FOV) also includes coronagraphy, medium-resolution $(\mathrm{R} \sim 5000)$ long slit spectroscopy at L/M and $\mathrm{N}$ band and polarimetry at N-band. The other main sub-system is the integral field high resolution spectrograph at $\mathrm{L} / \mathrm{M}(2.9-5.3 \mu \mathrm{m})$ band, with a spectral resolution of $\mathrm{R} \sim 100,000$. More information on the project overview and update on science cases can be found in the paper by Brandl et al. [1].

The METIS consortium consists of seven institutions from different countries: NOVA/Leiden University (the Netherlands), MPIA (Germany), CEA-Saclay (France), UK-ATC (United Kingdom), KU Leuven (Belgium), ETH Zürich (Switzerland), and the Universität Wien (Austria). The METIS consortium draws heavily from its long-term successful experience with numerous ground- and space-based infrared instruments, most recently the MIRI for the James Webb space Telescope (JWST).

*agocs@astron.nl; Tel +31 521595173

Advances in Optical and Mechanical Technologies for Telescopes and Instrumentation, edited by Ramón Navarro, Colin R. Cunningham, Allison A. Barto, Proc. of SPIE Vol. 9151, 91511L

(c) 2014 SPIE · CCC code: 0277-786X/14/\$18 doi: 10.1117/12.2055404 
In this paper we present innovative, immersed grating (IG) based optical designs for the SMO (Spectrograph Main Optics) module of the METIS high resolution spectrograph. In section 2 we outline the phase A design that contains a conventional echelle grating based configuration. In section 3 we present the IG itself and describe the IG based spectrometer design that was developed as part of a prototype immersed grating development project. In section 4 we present our novel, IG based spectrometer designs that are optimized in order to provide one of the following: best optical performance, best manufacturability, best packaging. In section 5 we explore a new test method that is ideally suited for some of the designs presented. In section 6, we propose a design, together with a MAIT plan that takes into account all lessons learnt from the different design and optimization processes.

\section{CLASSICAL ECHELLE BASED SPECTROMETER DESIGN}

A detailed description of the technical concept of METIS has been given in the papers by Lenzen et al. [2] and Kroes et al. [3]. The layout of METIS is shown in Figure 1 with the indication of the different sub-systems. The high resolution $\mathrm{L} / \mathrm{M}$ band spectrometer is an integral field spectrometer. The Phase A baseline design is based on a conventional echelle grating. Currently, the performance and feasibility of an immersed grating solution is being investigated and if the performance of the system proves superior it will be adopted as new baseline design. More information on the immersed grating development project can be found in the paper by Amerongen et al. [4].

\subsection{High resolution spectrometer}

The high resolution spectrometer should be operated in the $\mathrm{L} / \mathrm{M}$ bands, in a cryogenic environment $\left(<85^{\circ} \mathrm{K}\right)$ and it should provide a spectral resolution of 100000 at $4.65 \mu \mathrm{m}$. It is divided into two units, the Spectrometer Pre Optics (SPO) and the Spectrometer Main Optics (SMO). The SPO consists of the transfer optics and the integral field unit (IFU). The $\mathrm{SMO}$ is based on a conventional grating spectrometer, where the required order separation is achieved by a pre-disperser and a spatial filter.

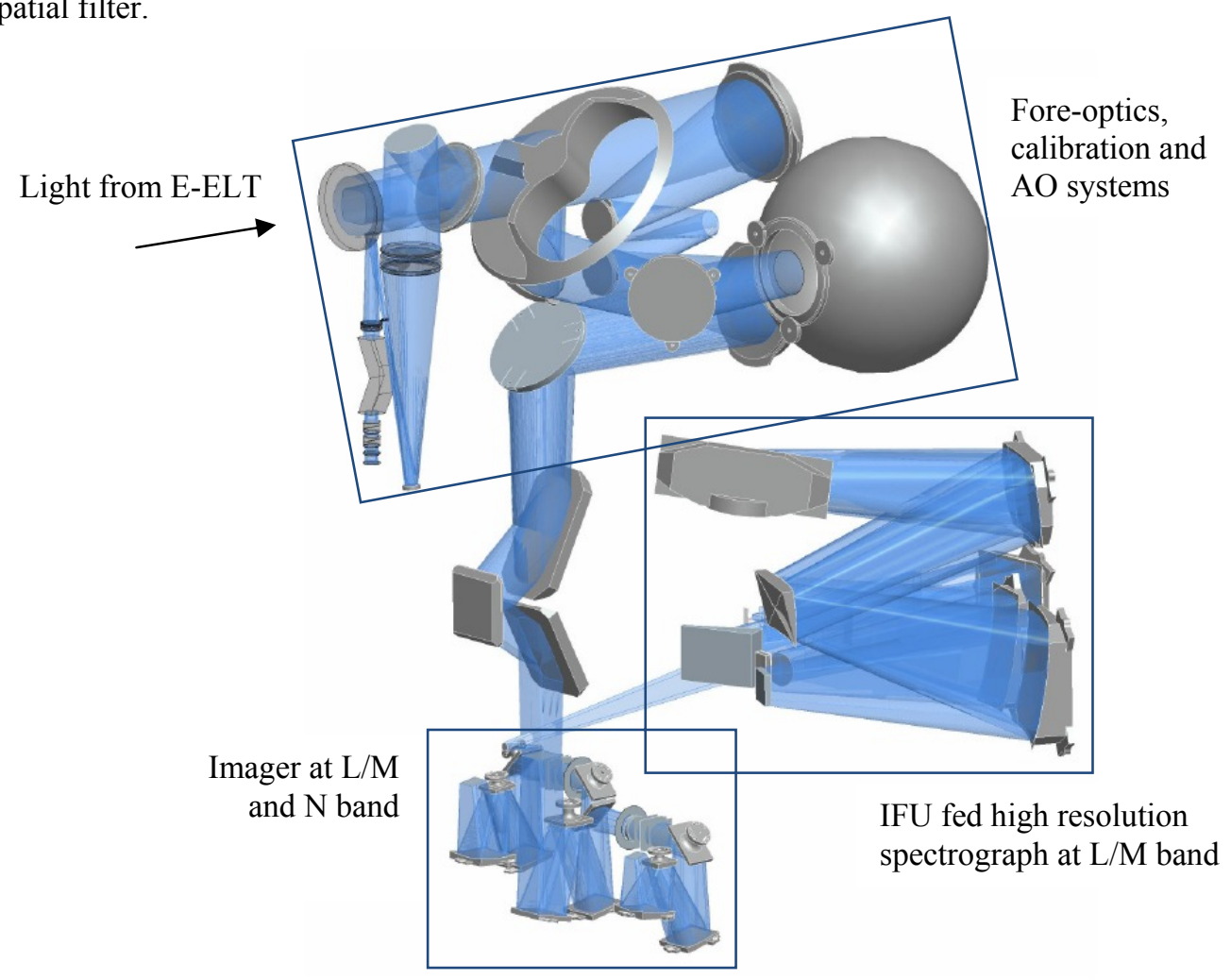

Figure 1 The layout of METIS is shown with the indication of the different sub-systems.

\subsection{The SMO and the classical grating}

The SMO has an F/10.3 telecentric input beam on a curved focal plane. Light is then collimated with an off-axis parabolic mirror and a ZnSe prism acts as a pre-disperser in the double pass configuration. A small wavelength band can 
be selected with a mask by rotating this prism. After the mask a pupil relay mirror couples the light from the predisperser into the main disperser. The latter is based on a double pass TMA, where the grating is positioned in a near Littrow configuration.

The grating itself has extremely challenging requirements, it should have very coarse ruling $(14.438 / \mathrm{mm}$ groove density), blaze of 65 degrees, large size $(400 \mathrm{~mm} \times 140 \mathrm{~mm})$ and maximum ghost level of $10^{-4}$. The WFE requirement is also stringent, around 100nm RMS, but somewhat flexible due to the possibility of redistributing WFE among the optical components in METIS. Given all these requirements, the fabrication of the classical echelle grating is an extremely challenging task. Only one or two manufacturers in the world would be able to supply the required grating and most of the parameters would need to be delivered on a best effort basis.

\section{CURRENT IG BASED SPECTROMETER DESIGN}

\subsection{The immersed grating}

Considering the difficulties of the manufacturing of the classical echelle grating proposed in phase A, it was decided to investigate the possibility to employ an IG in the heart of the spectrometer. Based on the wavelength range $(2.9 \mu \mathrm{m}-5.3$ $\mu \mathrm{m}) \mathrm{Si}$ is ideally suited as immersion material. Apart from the good transmission, single crystal Si also has beneficial anisotropic etching characteristics that allows for tailoring the groove profile to the specific grating requirements. The grating is obtained via etching into the $\mathrm{Si}$ with the surface cut along the $<100>$ crystal plane. The grooves thus will have a natural blaze angle close to 54.7 degrees depending on the anisotropic etch ratio. The angle of incidence on the grating will be close to this blaze angle. The grating is positioned at the bottom side of a Si prism (Figure 1). The grating is produced on a regular Si wafer that is bonded to the bottom surface of a Si prism. The light enters and exits the prism through the same facet.
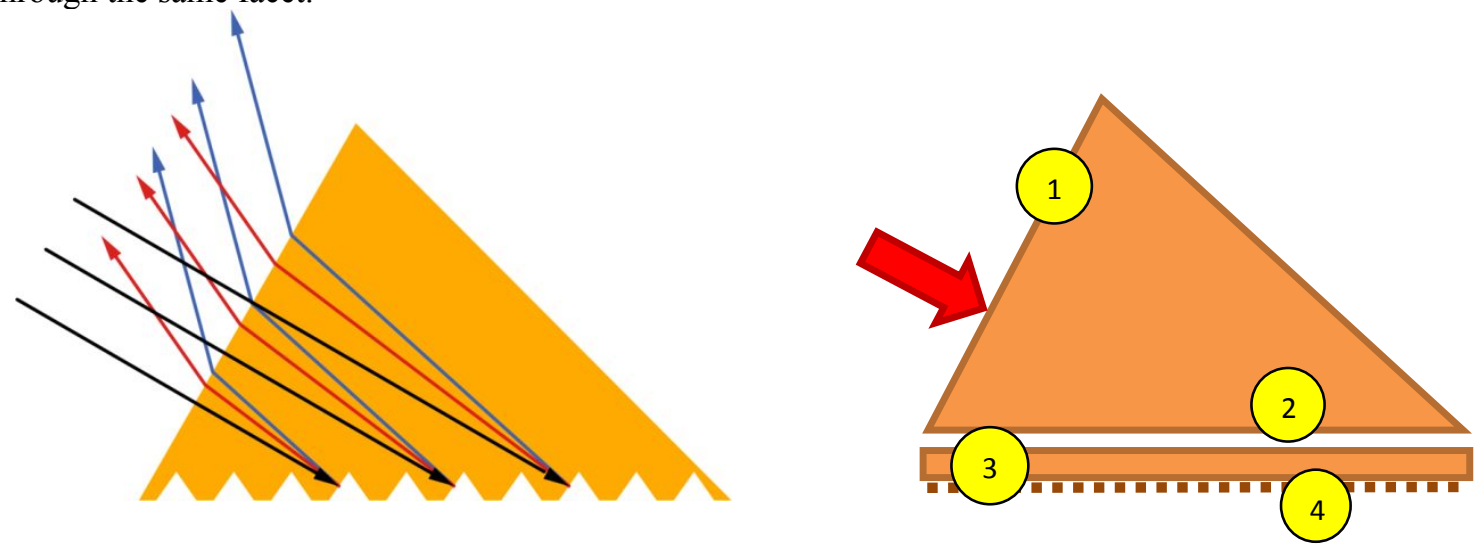

Figure 2 On the left the immersed grating principle is shown: light enters the prism through a polished entrance facet; after diffraction at the grating facet the dispersed light leaves the prism through the same facet. On the right the WFE contributors of the immersed grating are listed. The entrance surface is indicated with the arrow. \#1 is the WFE of the front surface of the prism. \#2 is the WFE of the back surface of the prism (before fusion of the prism with the wafer containing the grating). \#3 is the WFE that results from the wafer total thickness variation (TTV, before fusion with the prism) and \#4 is the WFE of the grating in reflection as measured from the air side of the grating. Material inhomogeneity is considered negligible.

\subsection{IG based design developed as part of the prototype immersed grating development project}

Figure 3 shows the optical layout of the relevant optical path of the IG based design that was developed as part of the prototype immersed grating development project. The pre-disperser configuration is the identical for the classical and IG based designs and it consists of a ZnSe prism and an Off Axis Paraboloid mirror. The optical system is the same double pass TMA configuration as for the phase A design, but the optical characteristics of the relay mirror, the TMA components and separations between the mirrors were re-optimized in order to minimize WFE on the detector. By using IG instead of the classical grating, the beam size can be reduced by $50 \%$ and the complete volume of the SMO (with mounts, mechanical structures, etc.) can be reduced by a factor of four. Less weight, less structure, smaller packaging and better stiffness are the important mechanical advantages of the IG based system. 
The greatest challenge for the IG is to meet the WFE requirement. In Figure 3 (right) the errors that contribute to the WFE budget of the IG are shown. \#1 and \#2 are the surface figuring of the front and back surfaces of the prism (before bonding and fusion of the prism with the wafer containing the grating), \#3 is the total thickness variation of the wafer and finally \#4 are the errors originate in the grating itself. The latter can be broken down into further four subgroups: line width variation after reactive ion etching, lithography distortion errors, lithography stitching errors and mask writing errors. Material inhomogeneity is not indicated, because for Si the material standard and properties as so extremely good that this error considered negligible.

In Figure 3 (right), the RMS WFE (in waves) vs. wavelength (in $\mu \mathrm{m}$ ) is shown for the current IG based spectrometer design. The WFE requirement, that the spectrometer be diffraction limited, is satisfied. Nevertheless, taking into account the above mentioned errors of the IG and additionally the tolerances of the optical components of the complete optical path, it is obvious that there is a need for a large margin in the WFE error budget.
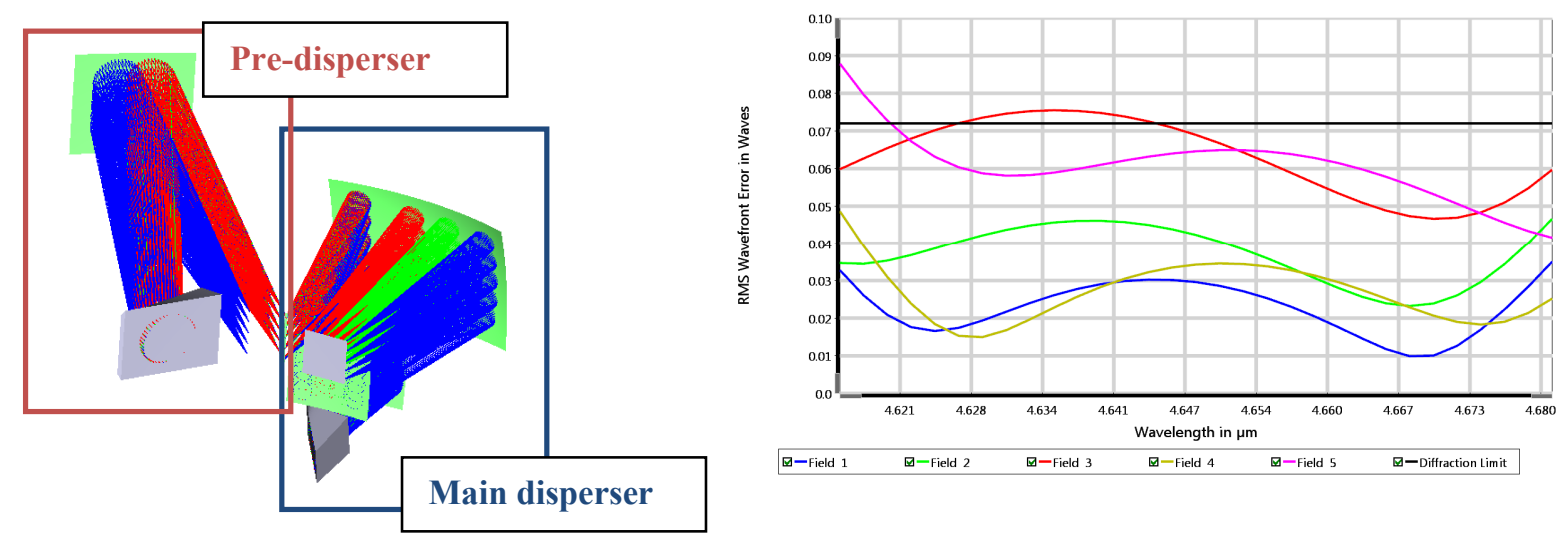

Figure 3 Layout is shown on the left for the IG based design that was developed as part of the prototype immersed grating development project. RMS WFE (in waves) vs. wavelength (in $\mu \mathrm{m}$ ) is shown on the right (the diffraction limit is indicated with a horizontal line).

\section{NOVEL SPECTROMETER DESIGNS}

In the following we present alternative IG based spectrometer designs. First we investigate how to improve the optical design with regards to WFE (4.1). Afterwards we explore the possibility of converting one or more TMA mirrors from off-axis conic sections into spherical mirrors (4.2). We do this in order to improve the manufacturability of the optical components. Thirdly, we describe spectrometer designs that allow better packaging and scattered light control (4.3). Finally we investigate systems in the context of testing and propose solutions for optical testing the complete spectrometer optical path in the warm (4.4).

\subsection{Optimizing for best optical performance}

We intended to improve the optical performance of the spectrometer by re-optimizing the optical design. The merit of our optimization was RMS WFE. We created an optimization method whereby the TMA has the maximum degrees of freedom (DOF) possible. During TMA optimization, the difficulty usually comes from the need to achieve an unobscured system, while maximizing optical performance. One solution is to carefully select the off-axis angle of the TMA field-of-view (FOV) and eccentricity of the pupil with respect to the axis of symmetry of the TMA (which is usually close to the axis of the $2^{\text {nd }}$ mirror). We improved the optical design by creating a 'floating TMA' model, so that these two parameters are actually variables and the mirrors have the possibility to 'float' around during optimization. Naturally, constraints had to be set in order to avoid obscuration by the mirrors, but knowing the pupil size, the plate scale on the detector and the approximate footprints on the mirrors, the limiting conditions can be set in a way that the system stays un-obscured. The result of the optimization is shown in Figure 5, where the WFE vs. wavelength is shown for two systems optimized in the way described. Comparing these figures with Figure 3 it is clear that a factor of 3 improvement in RMS WFE can be achieved. The optimization is done for two different TMA configurations shown in Figure 4. The TMA on the left has the pre-disperser mask (basically the image of the slit) and the detector next to each other, positioned along a plane perpendicular to the plane of the TMA mirrors. The TMA on the right has the mask and 
detector in the plane of the TMA mirrors. The latter configuration is advantegous because the distortion of the TMA can be used to compensate for the curved slit images on the detector, which is a consequance of the geometry of the rays arriving from the grating. The detector image with the slit geometry is shown in Figure 6 for the two cases. The other advantage of the 'in-plane mask and detector' design is that the detector is not perpendicular, but tilted with respect to the chief ray, which means that the ghost from the detector can be driven away from the incoming optical path. The disadvantage is that the dispersion direction is also in the plane of the mask and detector and so a more significant oversizing of the mirrors is needed in that direction, which can make packaging and scattered light control more difficult.

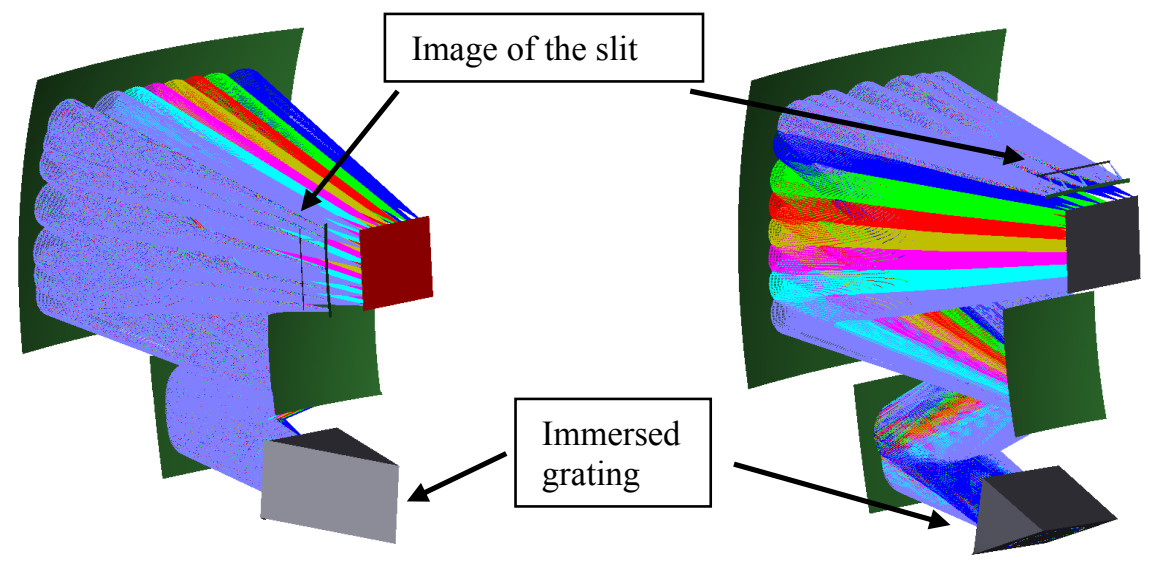

Figure 4 Layout of two new IG based designs. On the left the slit and the detector are next to each other on the right they are in the plane of the TMA mirrors.
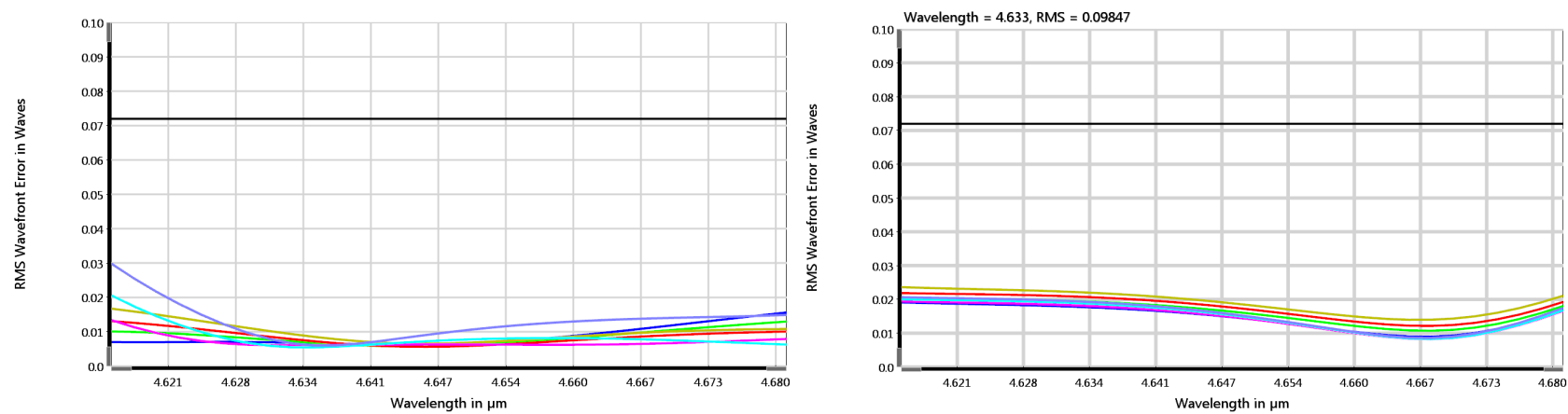

Figure 5 RMS WFE (in waves) vs. wavelength (in $\mu \mathrm{m}$ ) for the two design presented above. They are providing diffraction limited performance over the full field-of-view for the nominal wavelength range $(4.621 \mu \mathrm{m}-4.680 \mu \mathrm{m})$.
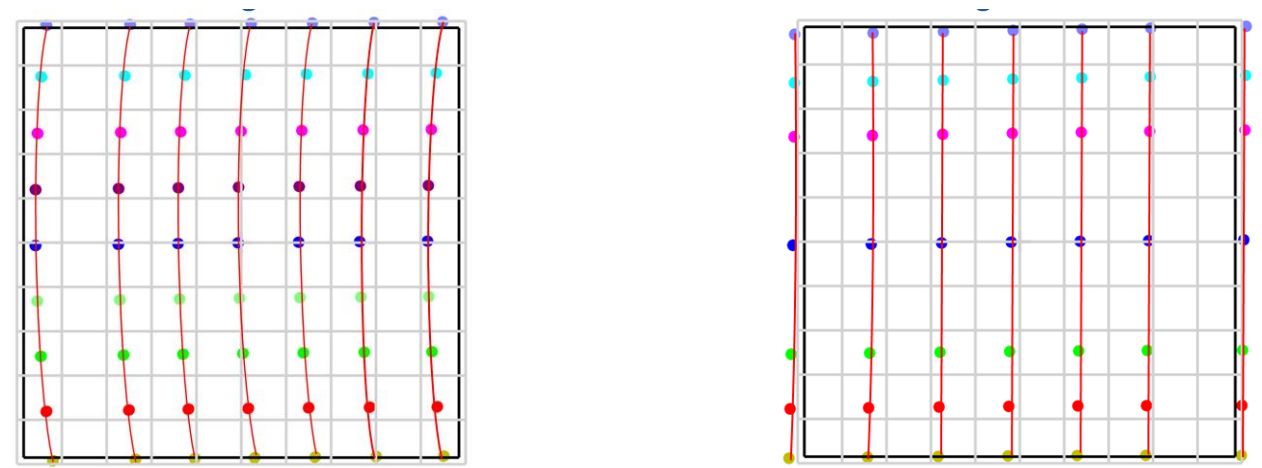

Figure 6 The slit geometry is shown for the two designs presented in Figure 3. By arranging the detector and the slit in the plane of the three mirrors of the TMA, straight slit geometry can be achieved. 


\subsection{Optimizing for best manufacturability}

In section 4.1 the re-optimization yielded very good optical performance, but the TMA mirrors were still off-axis conic sections, which are very difficult and costly to make, particularly in regard to surface figuring requirements $(15-25 \mathrm{~nm}$ RMS). In the present section we explore the possibility of converting one or more TMA mirrors from off-axis conic sections into spherical mirrors. We do this in order to improve the manufacturability of the optical components. Using the aluminium polishing expertise of NOVA, the spherical mirrors can be made in house and there is no need for complex and very expensive manufacturing process e.g. diamond turned aluminium mirrors with nickel plating and post polishing. Additionally, on these surfaces lower surface figuring errors can be achieved leading to a better balanced WFE budget. The optical system is re-optimized, again using the 'floating TMA' method described in the previous section and the result is depicted in Figure 7. Going from the current IG based design, the first step was already shown in the previous section, where three conics were still used. As a second step, the primary (largest) mirror of the TMA is transformed into a spherical mirror at the expense of some portion of the WFE. In this design the secondary and tertiary mirrors of the TMA are conic sections. Finally, the last re-optimization step produces a TMA with two spherical mirrors (primary and secondary) and the tertiary mirror is a freeform. The freeform surface consists of a Noll type Zernike polynomial with polynomials between $\mathrm{z} 4$ and $\mathrm{z} 11$. The surface deformations correspond to aberrations up to primary spherical aberration. More information on freeform mirror optimization can be found in [5] and [6].

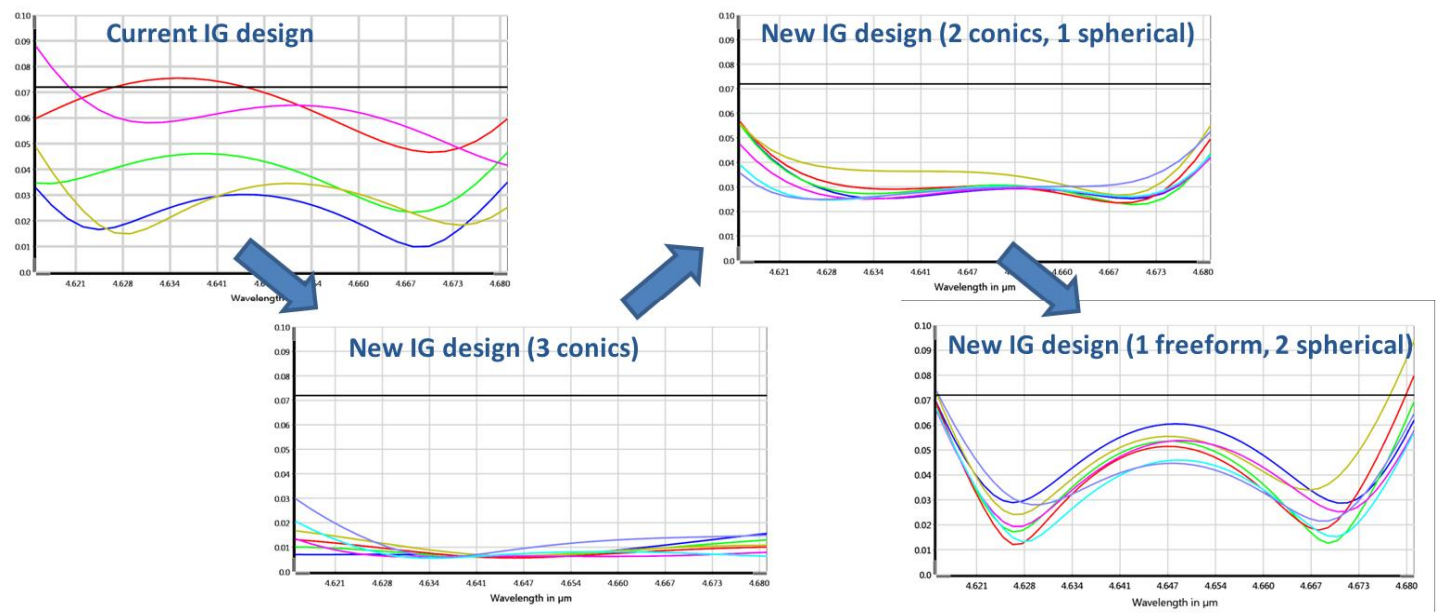

Figure 7 RMS WFE (in waves) vs. wavelength (in $\mu \mathrm{m}$ ) for four different SMO design, showing the evolution of the optical design process. Wavelength range shown is $4.621 \mu \mathrm{m}-4.680 \mu \mathrm{m}$.

In the following table two freeform based designs are compared. The first is the one described above, makes use of Zernike polynomials up to primary spherical aberration (z4-z11). The second design contains a freeform with Zernike polynomials between $\mathrm{z} 4$ and z22, which correspond to aberrations up to secondary spherical aberration. In both cases the Zernike polynomials are approximately centred on the (double pass) footprint on the tertiary mirror of the TMA. The table shows the WFE vs. wavelength and, as expected, the freeform with the higher degrees of freedom delivers better image quality. Looking at the residuals of the surface sag after tip, tilt and focus are subtracted (which also corresponds to the deviation from the best fit sphere), coma is dominating. If the coma is also subtracted, astigmatism will become visible. The freeform with higher order Zernike polynomials allows separate wavefront control of incoming and returning beams (double pass), which is why the overall WFE is better for this design. Looking at the last figure of the table it becomes apparent that after all the Zernike polynomials are subtracted up to the primary spherical aberration, the residuals create a convex and concave surface for the incoming and outgoing beams.

The manufacturing of the freeform mirror can be done by stress polishing or by diamond turning, nickel plating and post polishing. The presence of two spherical mirrors in the designs provides the opportunity of assigning a larger portion of the overall WFE to the freeform mirror, which opens up the possibility for other manufacturing techniques as well. The testing of the freeform surface would be done in the spectrometer itself as explained in section 4.4. 

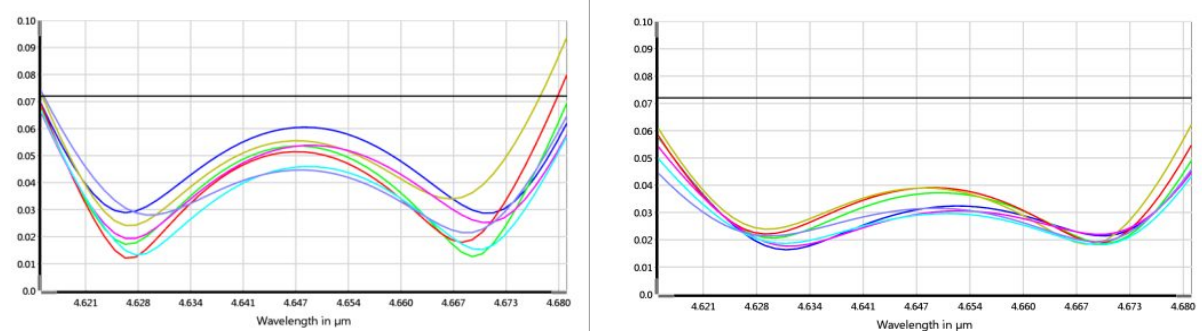

Figure 8 WFE (waves) vs. wavelength $(\mu \mathrm{m})$ for two freeform based spectrometer design. The design shown on the left has a freeform that consists of Zernike polynomials between z4-z11 (up to primary spherical aberration). The design on the right has a freeform that contains z4-z22 Zernike polynomials (up to the secondary spherical aberration).


Figure 9 Surface sag information is shown for the two designs mentioned above. In the top row surface sag residuals are plotted for the two designs after focus, tip and tilt are removed (basically the plot shows the deviation from the best fit sphere), left RMS $2.3 \mu \mathrm{m}$, right RMS $2.9 \mu \mathrm{m}$. In the middle row residuals are shown, after focus, tip, tilt and coma are removed, left RMS $1.0 \mu \mathrm{m}$, right RMS $1.1 \mu \mathrm{m}$. Finally residuals are plotted for the second design after Zernike polynomials are removed up to z11 (RMS $0.25 \mu \mathrm{m})$. Since the first design has Zernike polynomials up to z11, there are no residuals to show. 


\subsection{Optimized for best packaging}

We investigated optical designs that are favorable to the positioning and mounting of the optical components. Using the same optimization methodology, but changing the constraints and the possible space envelopes for the optical components, the mirrors could be separated significantly. In Figure 10 two TMA designs are shown with different mirror configurations. By separating the mirrors more in the vertical direction (in the plane of the mirrors), more space is available for mounting. The advantage of this type of TMA is that it is very compact; in some occasions it is also called Three Mirror Short (TMS). On the other hand, regarding stray light it does not perform very well, thus the proposed design with well-separated mirrors is also important regarding positioning baffles so that stray light can be kept under control.

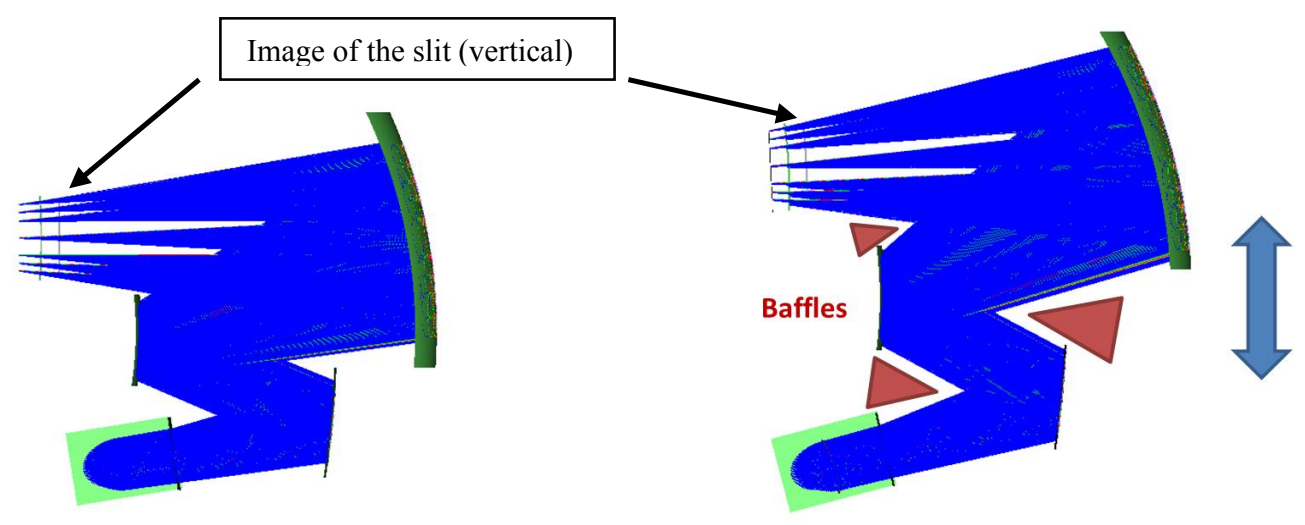

Figure 10 Two TMA designs are shown with different mirror configurations. The TMA on the right is preferable with respect to mounting the optical components and also regarding stray light control and baffling.

\section{TESTING}

Since the instrument operates in a cryogenic environment, the project phase of testing becomes extremely important. By thinking about Alignment, Integration and Testing (AIT) in early phases in the project, a lot of cost and effort can be saved later. Also, it is inevitable that reference surfaces, alignment targets, fiducial markers, etc. are identified in early phases and modelled in the mechanical design. Since the 'warm' and 'cold', or science and testing operation may require the use of different wavelength ranges, or even additional components in the optical path, the footprints on optical surfaces can be different and so the prescription of the clear aperture of optical surfaces have to be adjusted accordingly.

A new testing method was explored, which makes possible the testing of the complete optical path of the spectrometer main optics in the warm with visible light (e.g. $633 \mathrm{~nm}$ ). The main idea is that the immersed grating would be turned around in order to be able to use not in immersion, but in pure refection from the outside/wafer side. It has been modelled and the geometric spot size (only using ray tracing, not considering diffraction) is very similar for the nominal $4.65 \mu \mathrm{m}$ in immersion and for $0.633 \mu \mathrm{m}$ in reflection from the wafer side. Naturally, for the visible light a different diffraction order will produce 'close to Littrow' condition, but with a minor decentre of the detector the difference can be compensated for. If the pre-disperser is also included in the test, due to the dispersion of the ZnSe, a different prism rotation will be necessary that is outside of the range of rotation for science operation. Also, if not only monochromatic wavelength is used for the test, but, e.g. the full field of view on the detector ought to be tested, a larger pre-disperser mask and pupil relay mirror are needed. In principle, the substrate-removed Hawaii-2RG detector can be used in the visible ( $70 \%$ QE down to $400 \mathrm{~nm}$ ), so the same detector can be used for the proposed test, although probably it is worth having a test detector with smaller pixels. Due to the fact that diffraction limit scales with wavelength, the visible test can give a very good WFE measurement scheme, which would show the geometrical aberrations otherwise concealed by diffraction in the mid infrared science wavelengths.

The disadvantage of the testing method comes from the fact that a different WFE is measured in immersion compared with in reflection from outside. Consequently, prior to the proposed test, all the errors that contribute to the total WFE of the immersed grating have to be measured. Looking at Figure 2 again, the following considerations can be done. The prism front and back surfaces can be polished flat with very high accuracy and also they can be measured easily with an interferometer. The TTV of the wafer can be improved by wafer planarization with e.g. magnetorheological finishing 
(MRF) and reportedly $10 \mathrm{~nm}-15 \mathrm{~nm}$ RMS TTV were achieved. The grating patterning errors are the ones that can cause problems regarding the proposed testing method. Based on the experiences in the IG proptotype development project for METIS, the line width variation is the worst offender in the WFE budget. Based on the measurements, the line width variation was very close to linear, which produced astigmatism, when measured with an interferometer in reflection from the wafer side. The linear line width variation produces the same astigmatism from both sides of the grating, so in principle the proposed test method yields the same results as the science optical path. As a summary, generally very good (flat) surfaces are needed for the prism and the wafer and the only significant WFE contributor should come from linear line width variations. Based on the experiences with the prototype IG, this is the expected scenario, so the test method can be a viable scheme for AIT.

\section{A PROPOSED IG BASED DESIGN AND MAIT PLAN}

In Section 4 we developed models and investigated different designs in order to satisfy certain requirements, most of the time individually. In the following we propose a design, together with an MAIT plan that takes into account all the lessons learnt from the different designs and optimization processes. It is an immersed grating based system that contains pre-disperser and a spatial filter for order separation and a double pass TMA with the IG as main disperser. The TMA consists of spherical primary and secondary mirrors and freeform tertiary mirror. It has very good image quality and it satisfies the WFE requirements. By converting two TMA mirrors from off-axis conic sections into spherical mirrors, we improve the manufacturability of the optical components and at the same time achieve a better balanced WFE budget. The TMA mirrors are well separated, as a result more space is available for packaging optical components and for baffles, so that stray light can be kept under control.

We propose the following MAIT plan for the system mentioned above:

1. Manufacture the primary and secondary spherical mirrors and measure them.

2. Manufacture the silicon prism and wafer, measure surface figure of the prism on the front and rear sides, measure the TTV of wafer (errors \#1, \#2 and \#3 in Figure 2).

3. Measure the immersed grating in reflection from the outside/wafer side (error \#4 in Figure 2).

4. Confirm that all measured errors that are related to the immersed grating are appropriate for the warm test (so that the measurement results are the same within a certain WFE tolerance, when the IG is used in immersion or in reflection from the outside/wafer side).

5. Re-optimize the freeform surface in the optical model based on the measurent of the immersed grating and manufacture the freeform surface.

6. Align all components in the spectrometer.

7. Turn around the immersed grating, test the system in visible in the warm and measure performance.

8. Post polish freeform or remanufacture freeform, if necessary.

In the proposed MAIT, essentially, we intend to use the METIS spectrometer itself to test the freeform. In this way it is not necessary to design a separate test-setup for the manufacturing of the freeform surface. Also, by using the measurement of the IG, the possibility of the re-optimization of the freeform surface allows a looser WFE requirement for the immersed grating related to linear line width variation, which seems to be advantageous.

We modeled grating errors including linear line width variation and re-optimized the freeform surface in the proposed optical model. The results are shown in Figure 11. The image quality of the spectrometer could be recovered almost completely using the re-optimization of the freeform surface. In Figure 12 the surface sag of the freeform is shown before and after the re-optimization and the difference is also presented, which shows almost pure astigmatism. It makes sense, since the worst offender in the optical tolerance chain of the immersed grating is the linear line width variation, which produces astigmatic errors. The immersed grating is in the pupil and the freeform mirror is located very close to this pupil and it seems that it is capable of correcting the mentioned errors. 


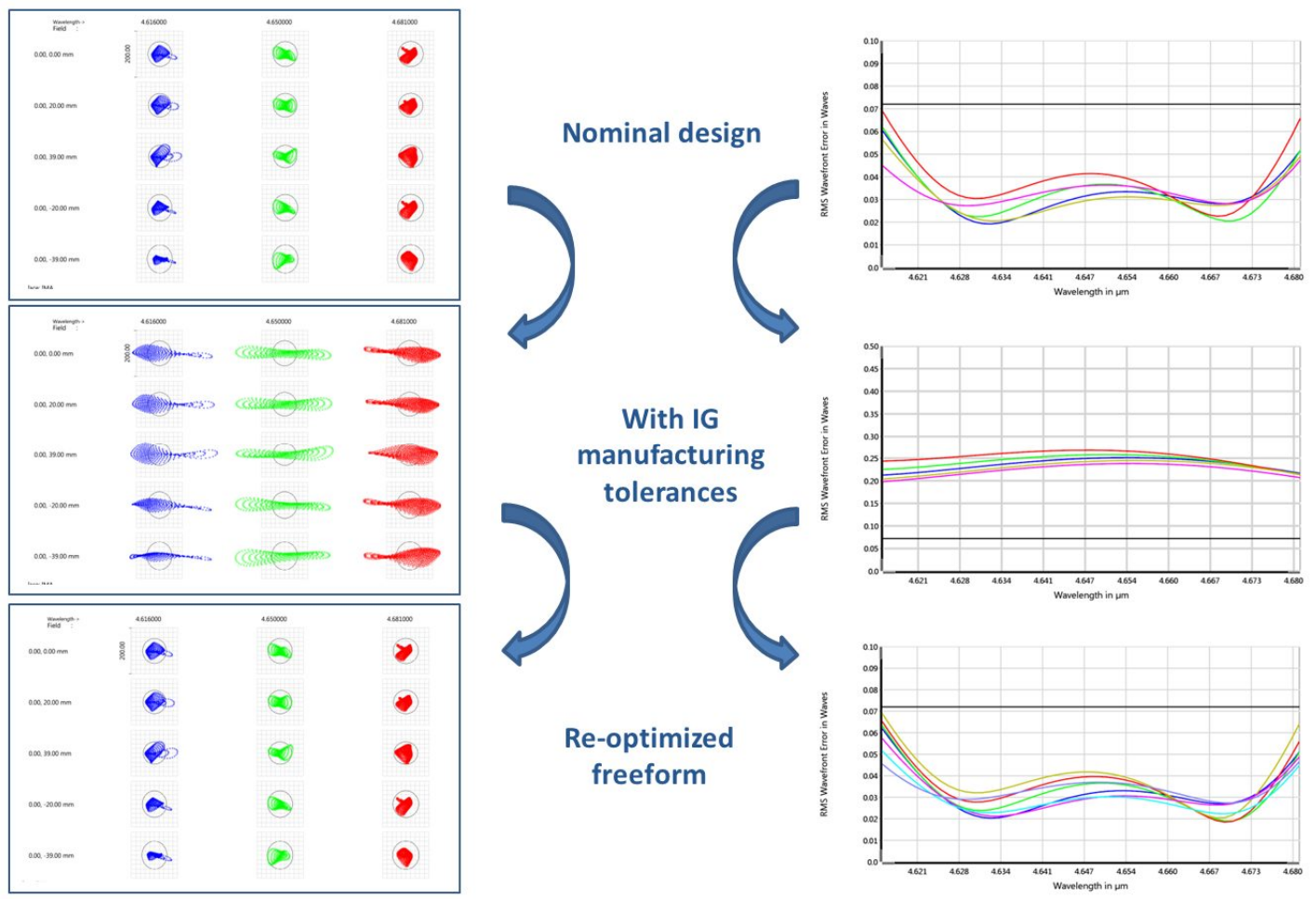

Figure 11 The re-optimization process of the freeform is shown above. The immersed grating errors are measured and based on the measurements, the freeform surface is re-optimized. The worst offender is the variable grating period that produces elongated spots. After re-optimization the image quality of the spectrometer is recovered.
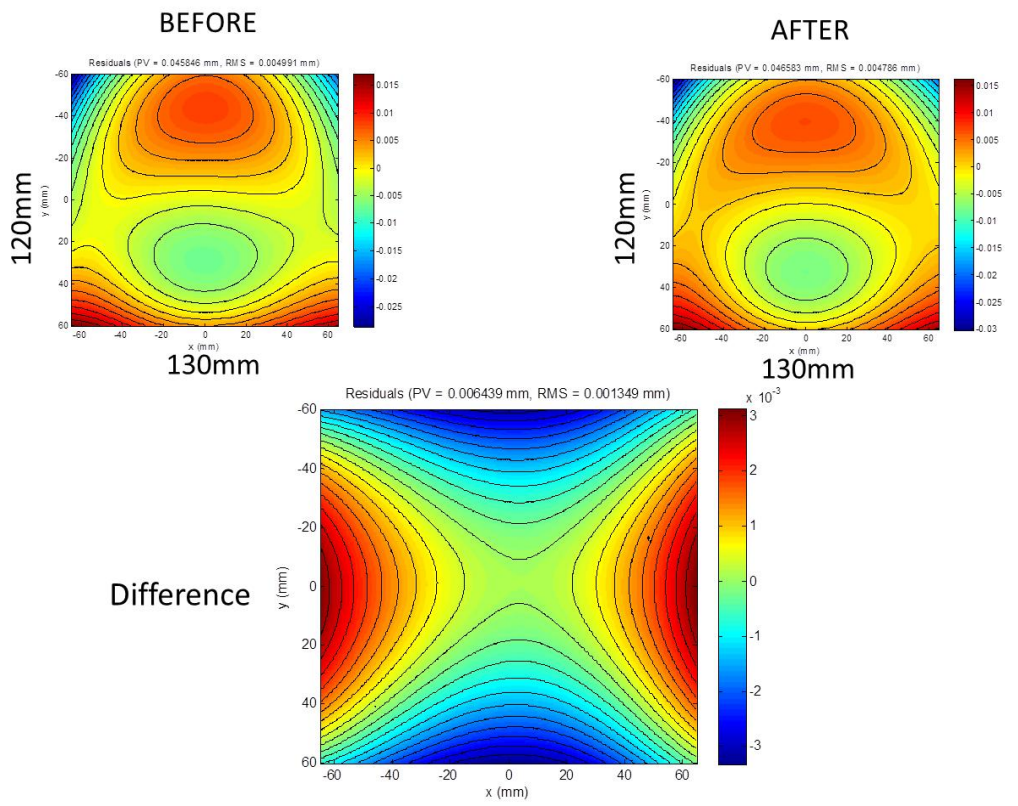

Figure 12 The re-optimization of the freeform surface results an almost pure astigmatism. It makes sense, since the worst offender in the optical tolerance chain of the immersed grating is the linear line width variation, which produces astigmatic errors. 


\section{CONCLUSIONS}

We presented innovative, immersed grating based optical designs for the SMO (Spectrograph Main Optics) module of the METIS high resolution spectrograph. Using novel optimization techniques we re-optimized the current double-pass Three Mirror Anastigmat design for best optical performance (WFE vs. wavelength), best manufacturability (converting off-axis mirrors to spheres) and best packaging (separation of the TMA mirrors). We investigated a test method that can be used at room temperature using visible wavelength. Based on experiences gained from all these exercises, we proposed a TMA that consists of spherical primary and secondary mirrors and freeform tertiary mirror. We also presented a complete MAIT plan that has several advantages, e.g. the use of the METIS spectrometer itself to test the freeform and even do iterative post polishing steps.

The optical systems and processes presented here will have to be developed further with more thorough analyses in order to decide whether the proposed solutions are applicable for the optical architecture of METIS.

\section{ACKNOWLEDGEMENTS}

The immersed grating development project has been made possible by Netherlands Organization for Scientific Research (NWO) via the ESFRI-project grant number 184.021.006. The freeform mirror development project is funded by the European FP7-OPTICON WP5 "Freeform Active Mirrors Experiment".

\section{REFERENCES}

[1] Brandl, B. R. et al., "METIS: the mid-infrared E-ELT imager and spectrograph,” Proc. SPIE 9147-73 (2014).

[2] Lenzen, R. et al., "METIS: System engineering and optical design of the mid-infrared E-ELT instrument," Proc. SPIE 7735-283 (2010).

[3] Kroes, G. et al., "METIS opto-mechanical design \& packaging study,” Proc. SPIE 7735-200 (2010).

[4] Van Amerongen, A. H. et al., "Development of silicon immersed grating for METIS on E-ELT," Proc. SPIE 8450-100 (2012).

[5] Agócs, T., et al., "Optimizing an active extreme asphere based optical system," Proc. SPIE 85501G (2012).

[6] Agócs, T., et al., "Freeform mirror based optical systems for FAME," Proc. SPIE 9151-90 (2014). 